\title{
HEPATIC INVOLVEMENT IN PATIENT WITH ASIA SYNDROME
}

\author{
Martos Ruiz V, Cascales Vallejo Al, García Cortacero E \\ Hospital Santa Ana. Motril, Granada.
}

\section{Resumen}

El nombre de síndrome autoinmune /inflamatorio inducido por adyuvantes (Síndrome ASIA o de Shonefeld) se relaciona con la exposición típicamente de alguna de estas sustancias: vacunas (especialmente papiloma humano), silicona, escualeno, hidróxido de aluminio, parafina líquida, acrilamidas, ácido hialurónico o metacrilato. Puede dar manifestaciones inflamatorias tanto locales como sistémicas. Presentamos el caso de una paciente con prótesis de silicona en mamas y tatuaje de piel y labios, en estudio por hepatitis de perfil colestásico, en la que aparecen lesiones cutáneas que se biopsian (granulomas no necrotizantes), siendo esto clave para el diagnóstico de reacción sarcoidítica.

Palabras clave: ASIA, Shoenfeld, hepatitis colestásica.

\section{Abstract}

The name autoimmune/inflammatory syndrome induced by adjuvants (ASIA or Shonefeld syndrome) is typically related to the exposure to one of these substances: vaccines (especially human papilloma), silicone, squalene, aluminum hydroxide, liquid paraffin, acrylamides, hyaluronic acid or methacrylate. It can present both local and systemic inflammatory manifestations. We present the case of a patient with silicone breast prosthesis and skin and lip tattoo, under study for hepatitis with a cholestatic profile, in which appear skin lesions that are biopsied (non-necrotizing granulomas), this being key to the diagnosis of sarcoidosis reaction.

Keywords: ASIA, Shoenfeld, cholestasic hepatitis.

\section{Introducción}

El nombre de síndrome autoinmune /inflamatorio inducido por adyuvantes (Síndrome ASIA o de Shonefeld) fue acuñado por primera vez en 2011 con el propósito de englobar un creciente número de patologías caracterizadas por una disregulación del sistema inmune innato y adquirido tras la exposición a algún adyuvante. Dicho síndrome se relaciona con la exposición típicamente de alguna de 
estas sustancias: vacunas (especialmente papiloma humano), silicona, escualeno, hidróxido de aluminio, parafina líquida, acrilamidas, ácido hialurónico o metacrilato ${ }^{1,5}$. Puede dar manifestaciones inflamatorias tanto locales como sistémicas. Los nódulos inflamatorios localizados y paniculitis se observan hasta en el $88 \%$ de pacientes, y pueden evolucionar en una variedad de trastornos como cirrosis biliar primaria, Sd. Sjögren, sarcoidosis, enfermedad humana por adyuvantes, vasculitis, enfermedad inflamatoria intestinal, y polirradiculopatía inflamatoria ${ }^{2}$.

\section{Caso clínico}

Presentamos el caso de una paciente de 46 años con antecedentes personales de consumo de 2-3 cigarrillos/día, e intervenida por colocación de 2 prótesis mamarias e infiltración con hialurónico y tinta roja en labios, también tatuada en la espalda.

Acude a servicio de urgencias de nuestro hospital por cuadro de ictericia indolora de unas 4 semanas de evolución, con disconfort en hemiabdomen superior. En analítica se observa una hepatitis de predominio citolítico (Bilirrubina total $5,3 \mathrm{mg} / \mathrm{dL}$, GOT $874 \mathrm{U} / \mathrm{L}$, GPT 1088 U/L, FA 89 U/L, Actividad protrombina 71\%, INR 1. 33); por dicha coagulopatía se decide ingreso en unidad de Digestivo.

Durante su estancia en planta, aparecen nódulos subcutáneos supraciliares y en labios (Figuras 1 y 2), motivo por el que consultamos con Medicina Interna. Llaman la atención varios detalles: 1) que unas semanas antes, se realiza tatuaje previo con tinta roja de contorno labial con aparición de progresivas lesiones nodulares violáceas pruriginosas en labio superior (al menos inicia 4 meses antes) y a nivel supraciliar (Figuras 1 y 2); 2) presenta pápulas de 1-2 mm en rodillas y 3) y tatuaje que tanto a visión directa como a la palpación está en relieve (Figura 3).

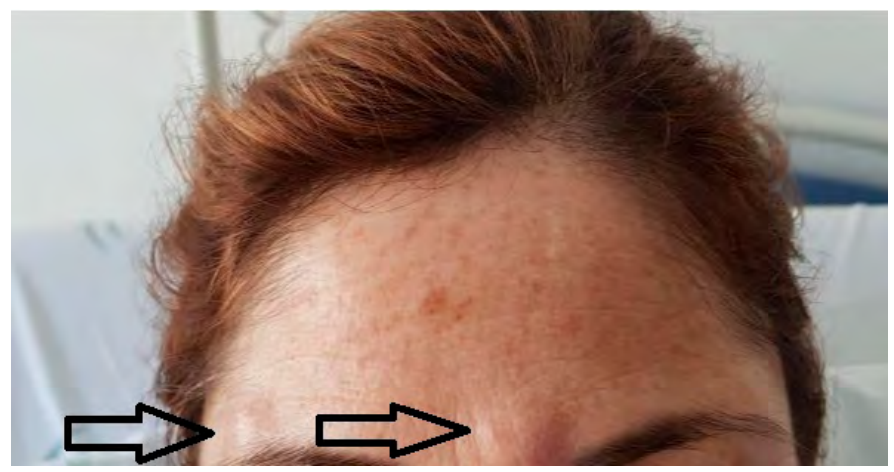

Figura 1

Nódulos supraciliares.

- Serologías y autoanticuerpos: AntiLKM, antimúsculo liso, Anticitrulina, ANA, ac antimitocondriales, anti-cardiolipina, anti-b-2 glicoproteína, antimieloperoxidasa y anti-proteinasa 3 NEGATIVOS. Serologías virales (VHB, VHA, VIH, VHC, VEB, VVZ, CMV, VIH negativos). Ceruloplasmina normal. Enzima convertidora angiotensina (ECA) normal.

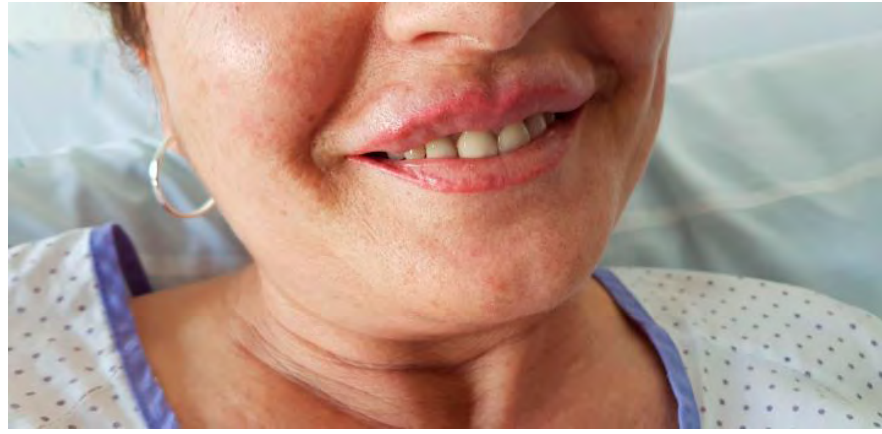

Figura 2

Nódulos labiales

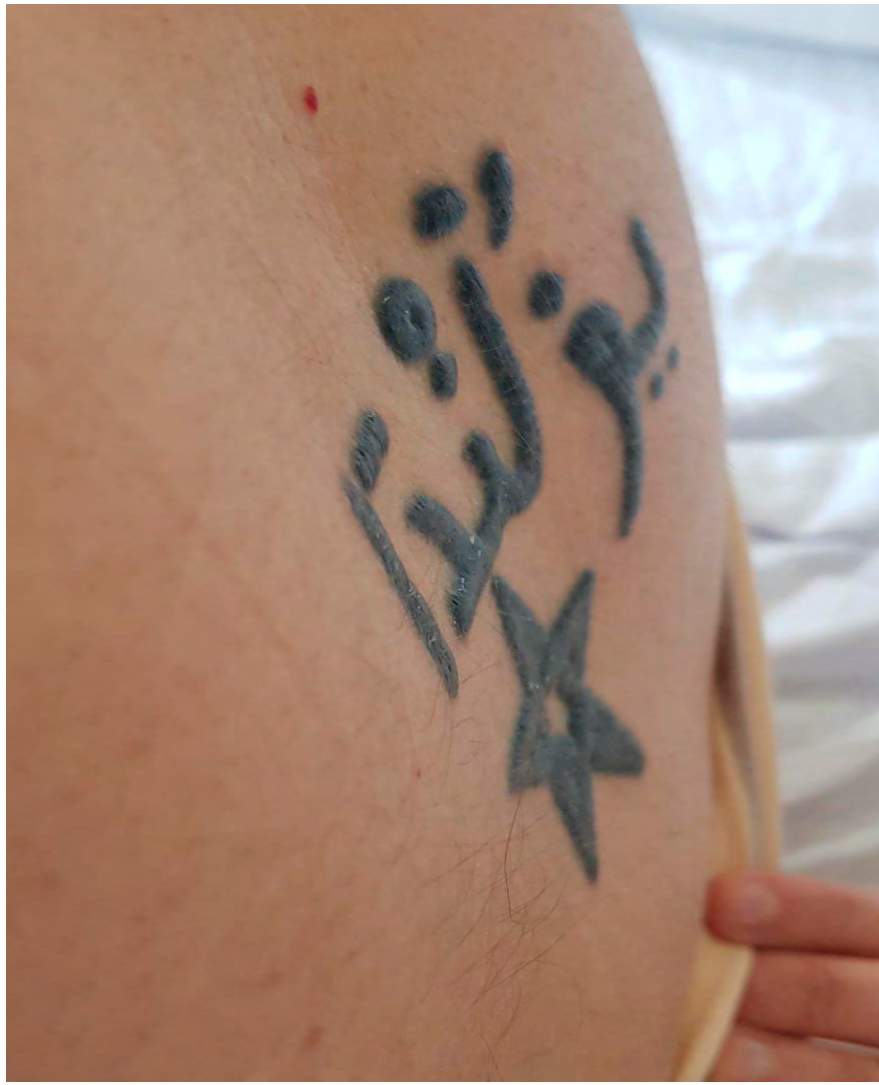

Figura 3

Tatuaje en relieve por infiltración sarcoidótica.

- TAC tórax, en el que se aprecian - Adenopatías hiliares bilaterales y mediastínicas y micronódulos cisurales bilaterales que podría estar en relación con afectación ganglionar y parenquimatosa por proceso granulomatoso tipo sarcoidosis.

- Ecografía mamaria: En la ecografía de mama izquierda objetivamos una irregularidad de los contornos de la prótesis mamaria con líneas hiperecogénicas en el interior de la prótesis. A nivel axilar objetivamos adenopatías hiperecogénicas de diverso tamaño, siendo la mayor de $30 \times 14 \mathrm{~mm}$ de diámetro, con sombra posterior típica de siliconomas, imagen de probable rotura extracapsular de prótesis de mama izquierda. 
- Biopsias lesiones cutáneas (nódulos faciales, pápulas en rodillas y tatuaje en zona sacra): TODAS presentan granulomas no necrotizantes. Negativo para hongos y TBC. En la biopsia hepática se observa intensa proliferación ductulillar con moderada inflamación inespecífica, con muy escasas células plasmáticas, no se observa parénquima hepático normal, no se identifican granulomas. Estos hallazgos pueden ser concordantes con una Colangitis biliar primaria estadio $1^{5}$. También podrían darse en el caso de una necrosis hepatocitaria de tiempo de evolución.

Se decide tratamiento con Prednisona $30 \mathrm{mg} /$ día e Hidroxicloroquina $200 \mathrm{mg} / 12 \mathrm{~h}$, calcio y colecalciferol y exéresis de las prótesis mamarias. Durante unos meses la paciente bajó la dosis de corticoides a 7,5 mg de prednisona por decisión propia, con ascenso de enzimas hepáticas, por lo que hubo que subir de nuevo a $15 \mathrm{mg} /$ día.

\section{Discusión}

En una revisión de una serie española de 46 pacientes, ${ }^{3}$ diagnosticados de síndrome Asia, se encontraron nódulos inflamatorios 40/45 (88\%), así como patologías tales como cirrosis biliar primaria, Sd Sjögren, sarcoidosis, enfermedad inflamatoria intestinal y polirradiculopatía. 5(11\%) se presentaron primariamente con una enfermedad autoinmune sistémica. Los biomateriales y prótesis pueden provocar enfermedades autoinmunes de aparición tardía, cumpliendo los criterios ASIA, o presentándose primariamente como reacciones locales/inflamatorias que pueden eventualmente evolucionar en trastornos autoinmunes sistémicos o granulomatosos que serían catalogados como ASIA2.

Los principales biomateriales usados suelen ser rellenadores faciales y para aumento de pecho. Interesantemente, el tiempo medio transcurrido antes de la manifestación clínica, puede ser hasta 70 meses (la paciente se había implantado las prótesis hace años). Los rellenadores faciales semi-permanentes, con un efecto de entre 6-12 meses podrían ser responsables de manifestaciones mucho después. Este hecho puede ser debido a un segundo efecto desencadenante de otros adyuvantes, particularmente otros pigmentos faciales, infecciones clínicas o subclínicas, traumas locales o vacunas. En conclusión, en un huésped predispuesto, 2 o más diferentes estímulos -como vemos en el caso de esta paciente que es portadora de una prótesis de silicona rota, un tatuaje en la espalda, y una reciente pigmentación con tinta roja labial ${ }^{2}$ - podrían manifestar reacciones autoinflamatorias sistémicas.

\section{Bibliografía}

1. Luis J. Jara, Grettel García-Collinot, Gabriela Medina et al. Severe manifestations of autoimmune syndrome induced by adjuvants (Shoenfeld's syndrome). Immunol Res 2017;65:8-16.

2. Balk EM, Earley A, Avendano EA, Raman G. Long-term health outcomes in women with silicone gel breast implants: a systematic review. Ann Intern Med 2016;164:164-75

3. Jaume Alijotas-Reig, Enrique Esteve - Valverde, Natalia Gil-Aliberas, Víctor García- Gimenez. Autoimmune/inflammatory syndrome induced by adjuvantsASIA- related to biomaterials: analysis of 45 cases and comprehensive review of the literature. Immunologic Research. 2018;66:120-140.

4. Ludwig J, Dickson ER, McDonald GS. Staging of chronic nonsuppurative destructive cholangitis (syndrome of primary biliary cirrhosis). Virchows Arch A Pathol Anat Histol 1978; 379:103.

5. Abdulla Watad, Vered Rosemberg, Shmuel Tosano, Jan Willem Cohen et al. Silicone breast implants and the risk of autoimmune/rheumatic disorders: a real- world analysis. International Journal of Epidemiology. 2018; 1846-1854. 